\title{
2 (S)TEM structural and compositional nanoanalyses of chemically 3 synthesized glutathione-shelled nanoparticles
}

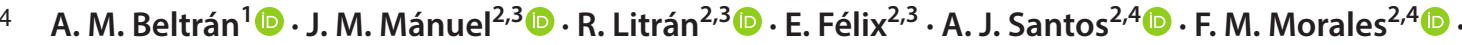 \\ 5 O. Bomatí-Miguel ${ }^{2,3}$ (D)
}

6

Received: 11 March 2020 / Accepted: 15 April 2020
○ King Abdulaziz City for Science and Technology 2020

\section{Abstract}

This work is focused on the characterization by transmission and scanning-transmission electron microscopy-related techniques of core-shell nanoparticles synthesized via chemical methods. Different semiconducting, pure metallic or oxide materials have been utilized as the core (cadmium telluride, gold, magnetite, or magnetite covered with gold) of the nanoparticle, while they have been, in all cases, functionalized by a thin amorphous glutathione layer, with the goal of using the nanoparticles in biomedical applications such as biomarkers, and computerized tomography and image magnetic resonance contrast agents. The results show that it is possible to visualize the glutathione layer using spectroscopic and imaging techniques, associated with electron microscopy (such as energy-dispersive X-ray spectroscopy and high-resolution transmission electron microscopy images), that this layer is present at the surfaces of all observed nanoparticles, and that it is no thicker than a few nanometers. Electron microscopy also revealed that the nanoparticles core is crystalline and, in average, around 5-nm size.

\section{Keywords Nanoparticle · Glutathione · Gold · Magnetite - QD · Electron microscopy}

\section{Introduction}

Nanoparticles (NPs) are defined as solid aggregates of atoms which, having different shapes, present dimensions between 1 and $100 \mathrm{~nm}$ (Hochella 2002), therefore, they can be considered a subgroup among the colloidal particles. Although first described during the Royal Society Bakerian Lecture of 1857, by Michael Faraday, on the interactions between light an metals, NPs have been always present in nature and

$\bowtie$ J. M. Mánuel

jose.manuel@uca.es

1 Department of Ingeniería y Ciencia de los Materiales y del Transporte. Escuela Politécnica Superior, Universidad de Sevilla, 41011 Sevilla, Sevilla, Spain

2 IMEYMAT: Institute of Research on Electron Microscopy and Materials, University of Cádiz, 11510 Puerto Real, Cádiz, Spain

3 Department of Condensed Matter Physics, Faculty of Sciences, University of Cádiz, 11510 Puerto Real, Cádiz, Spain

4 Department of Materials Science and Metallurgic Engineering, and Inorganic Chemistry, Faculty of Sciences, University of Cádiz, 11510 Puerto Real, Cádiz, Spain the human being have been using them, without a clear conscience of their existence, for centuries (Griffin et al. 2018). Nevertheless, their artificial synthetization, with the purpose of targeting specific applications, only started in the second half of the twentieth century (Birrenbach and Speiser 1976; Kreuter 2009). The use of NPs is extensive and they are employed to develop novel environment-friendly techniques, such as chemical mechanical polishing slurries with different metals (Zhang et al. 2019, 2020), machining methods (Zhang et al. 2015), and diamond wheels (Zhang et al. 2012). Thanks to these technologies, ultra-smooth surfaces and high-performance devices are achieved for several applications, i.e., semiconductor, microelectronics, and aerospace industries, which is extremely difficult to be fabricated by traditional machining and manufacturing (Zhang et al. 2017). The small size of these materials causes effects such as quantum confinement, which, in combination with their high reactivity due to a high specific surface area, makes NP engineering an ideal platform for custom-made functions, providing solutions for a large amount of disciplines, from electronics (Matsui 2005) to biomedicine (Karley et al. 2011; Ventola 2017; Gheibi Hayat and Darroudi 2019).

Among a large variety of compositions, three types of NPs present interesting features for biomedical applications:

\begin{tabular}{|l|l|l|l|l|}
\hline Journal : Large 13204 & Article No : 1418 & Pages : 7 & MS Code : 1418 & Dispatch : 24-4-2020 \\
\hline
\end{tabular}


gold (Au) NPs, quantum dots (QDs), and magnetic NPs. $\mathrm{Au}$ NPs have been widely utilized during the last decade in medical applications such as drug delivery and diagnosis (Kumari et al. 2019; Ramalingam 2019), used as computerized tomography (CT) contrast agents, and even as therapeutic agents in optical hyperthermia. The physical, chemical and optical properties of Au NPs, like their surface plasmon band (Amendola et al. 2017) and their capability to enhance the X-ray attenuation and their low cytotoxicity, make them highly attractive to biomedical uses. Magnetite $\left(\mathrm{Fe}_{3} \mathrm{O}_{4}\right)$ is a highly interesting material to utilize as NP since it is superparamagnetic at room temperature, and, when certain coating is used, its toxicity is reduced, even eliminated. Therefore, these types of NPs are ideal contrast agents for medical diagnosis via magnetic resonance (MRI). On the other hand, semiconductor QDs, such as cadmium telluride (CdTe) NPs, offer an interesting tool for biomarkers due to their unique size-tunable optical properties and, consequently, their sizedependent fluorescence (Le Trequesser et al. 2013). In this case, since the toxicity might also be a concern for these NPs, they are mostly utilized in ex vivo biomedical diagnosis (Kairdolf et al. 2013).

These systems offer another advantage: NPs can be functionalized and coated with a polymer layer in the sense of achieving biomedical applications, such as targeted drug deliver, or marker for imaging certain types of cells, thanks to a high, specific affinity of such polymer. Glutathione $\left(\mathrm{C}_{10} \mathrm{H}_{17} \mathrm{~N}_{3} \mathrm{O}_{6} \mathrm{~S}\right.$, or $\left.\mathrm{GSH}\right)$ is a tripeptide containing a thiol group to be linked to the NP surface, as well as both, amino and carboxylic groups that remain free to be subsequently crosslinked with other biomolecules, as we have previously shown (Hong et al. 2006; Kumar et al. 2014). In this work, we have used GSH as capping agent for the functionalization of four types of NPs: CdTe QDs with potential applications as biomarkers, gold NPs with potential applications as CT contrast agent, magnetite NPs with potential applications as MRI contrast agent, and, finally, gold-magnetite NPs than can be used as dual contrast agents for bimodal MRI and CT bimodal imaging. In all cases, NPs are "in situ" functionalized during the synthesis step, using GSH. Achieving a correct functionalization process is one of the most important goals for potential medical applications that require subsequent crosslinking with specific biomolecules.

It has to be taken into account, nevertheless, that, if the GSH layer is not properly linked to the NP, undesired results (such as the formation of a non-homogenous or a too-thick GSH layer) could produce defective NP systems. Therefore, to ensure that a proper synthetization has been achieved, a complete set of analyses have to be carried out. Such analyses should not only include chemical techniques, but also methods that provide reliable information on the distribution of GSH around the NP. Direct visualization of hydrophilic molecules in colloidal NP coating layers is possible through the use of high-cost equipment and complex analyses, such as last-generation electron microscopes or cryogenic-transmission electron microscopes. For this reason, researchers on the colloidal NP field commonly chose techniques that yield information on the electric charge of the NPs (such as measurements of zeta potential and isoelectric $\mathrm{pH}$ ) or on the composition of the coating layers (such as Raman and X-ray photoelectron spectroscopies). However, those methods do not reveal the thickness or the homogeneity of these layers around the NP. Thus, in this work, transmission and scanning-transmission electron microscopy, or (S)TEM, has been chosen as the main tool for the NP-GSH system since it comprehends techniques that join the structural and compositional characterization, with high spatial resolution. Precisely, this is the emphasis of the study: to demonstrate the usefulness of the combination of (S)TEM techniques to properly characterize the GSH layer on NPs. In this sense, in this work, three different GSH-covered NP systems are fully characterized by the use of this microscopy. Since the goal of this work is the characterization of GSH-covered NPs, these systems do not have by themselves a specific bio-functionalization; the carboxylic and amino terminal groups in GSH will allow subsequent crosslinking with biomolecules.

\section{Materials and methods}

The nanoparticles of this work, which are schematically illustrated in Fig. 1, were synthesized through different chemical methods and consist of four types of core materials, which are, in all cases, covered by a thin GSH layer. Later on, sets of nanoparticles were prepared and labeled,

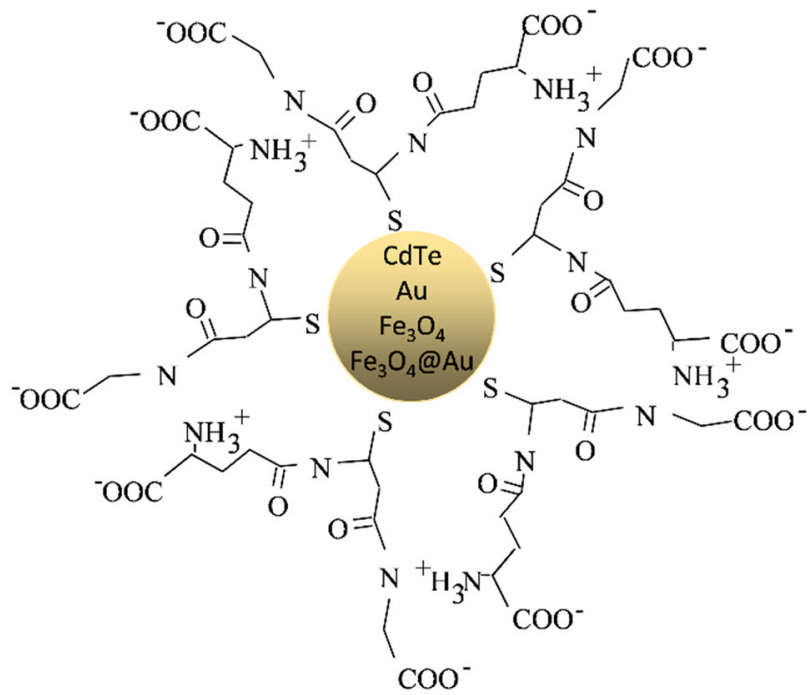

Fig. 1 NP concept, consisting of a core made from one out of four possible materials, and glutathione molecules covering it

4

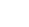

6

7

8

9

1

2

4

5

7

8

9

1

2

4

5

6

7

.

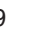

.

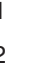

\begin{tabular}{|l|l|l|l|l|}
\hline Journal : Large 13204 & Article No : 1418 & Pages : 7 & MS Code : 1418 & Dispatch : 24-4-2020 \\
\hline
\end{tabular}


according to these core materials, as samples $\mathrm{S} 1-\mathrm{S} 4$, which were afterwards characterized by means of electron microscopy. In this sense, the label S1 indicates a sample of GSHcapped CdTe NPs. The material under the GSH layer in sample S2 particles is magnetite. The label S3 is used for the sample in which the core is solely formed by gold. Finally, sample S4 consists in threefold-material particles: an inner $\mathrm{Fe}_{3} \mathrm{O}_{4}$ core underneath a thin $\mathrm{Au}$ interlayer and a glutathione shell at the surface of the NP.

\section{Synthesis of samples}

A brief description of the chemical methods to obtain each sample is described hereafter. These methods are presented in more detail in a different work by the authors focused in the application of these materials as biomarkers for medical purposes (Beato-López et al. 2012, 2017; Fernández-Ponce et al. 2018). Commercial GSH was utilized to obtain the coated NP. In all cases, NPs were synthesized at the facilities of the Department of Condensed Matter Physics, associated with the Institute of Research on Electron Microscopy and Materials (IMEYMAT), of the University of Cádiz.

\section{Synthesis of GSH-capped CdTe NPs (sample S1)}

The synthesis of GSH-CdTe QDs was carried out in two steps, by introducing substantial changes on the methods shown in previous reports (Beato-López et al. 2012, 2017). First, tellurium $(\mathrm{Te})$ powder was reduced in the presence of $\mathrm{NaBH}_{4}$, to obtain the Te precursor for QD preparation. The GSH-CdTe QDs were prepared by a co-precipitation reaction. For this purpose, $0.19 \mathrm{~g} \mathrm{GSH}$, used as a stabilizing agent, and $0.046 \mathrm{~g} \mathrm{CdCl}_{2}$, used as the cadmium (Cd) precursor, were dissolved in $100 \mathrm{ml} \mathrm{MQ}$ water. The $\mathrm{Cd}^{2+}$ :NaHTe:GSH molar ratio was $4: 1: 10$. To promote the formation of $\mathrm{Cd}^{2+}-\mathrm{GSH}$ complexes at the QD surface, $\mathrm{pH}$ was adjusted to 8 by dropwise addition of a $1 \mathrm{M} \mathrm{NaOH}$ solution, under vigorous stirring. The obtained solution was placed in a 500-ml three-necked flask, where $2 \mathrm{ml}$ of fresh NaHTe (previously prepared in the first step) was added. The solution was heated under reflux in a nitrogen atmosphere. The final QD sizes can be controlled increasing temperature and reaction time.

\section{Synthesis of GSH-capped magnetite (sample S2)}

The synthesis of $\mathrm{GSH}-\mathrm{Fe}_{3} \mathrm{O}_{4}$ NPs was also performed in two steps. The first step consists of the preparation of a $\mathrm{NaOH}$ solution that was used as oxidizing agent for the coprecipitation of $\mathrm{Fe}$ and formation of $\mathrm{Fe}_{3} \mathrm{O}_{4}$. This solution was prepared by heating $20 \mathrm{~g}$ of $\mathrm{NaOH}$ and $20 \mathrm{ml}$ of diethylene glycol at $120^{\circ} \mathrm{C}$ for $1 \mathrm{~h}$. After this time, the resulting solution is stored at $70{ }^{\circ} \mathrm{C}$ for its posterior utilization. The second step was the main thermal decomposition and coprecipitation reaction. For that, $0.55 \mathrm{~g}$ of $\mathrm{FeCl}_{3}$, used as iron (Fe) precursor, has been dissolved in $15 \mathrm{ml}$ of diethylene glycol solvent. The obtained solution has been placed in a 500-ml three-necked flask, where the GSH used as capping agent has been added. The solution was heated to $200{ }^{\circ} \mathrm{C}$ under reflux in a nitrogen atmosphere to promote the thermal decomposition of Fe salt. After heating for $30 \mathrm{~min}$, the $\mathrm{NaOH}$ solution previously prepared had been added to provoke the co-precipitation of $\mathrm{Fe}$ and the formation of iron oxide.

\section{Synthesis of GSH-capped Au NPs (sample S3)}

$\mathrm{Au}$ NPs were prepared by the reduction of $10 \mathrm{ml}$ of 0.01 $\mathrm{MHAuCl}_{4}$ mixed with $10 \mathrm{ml} 0.026 \mathrm{M} \mathrm{GSH}$. The mixture was vigorously stirred for 30 min under a nitrogen atmosphere. Then $\mathrm{NaBH}_{4}$ was added dropwise at 1:5 $\mathrm{HAuCl}_{4} / \mathrm{NaBH}_{4}$ molar ratio. After 30 additional minutes, the red wine color solution was stored at $8{ }^{\circ} \mathrm{C}$ protected from light. All of them were aqueous solutions.

\section{Synthesis of GSH-Au magnetite (sample S4)}

In this case, GSH-magnetite was added to a solution containing $\mathrm{HAuCl}_{4}$ and $\mathrm{GSH}$ in excess to form the Au core over the NPs previously prepared.

\section{Characterization techniques}

Structural and compositional characterizations of all samples have been performed using different (S)TEM-related techniques. Imaging methods such as conventional bright field (BF-TEM), high-resolution (HRTEM) and high-angle annular dark field STEM (HAADF-STEM) allowed to visualize structural features, such as size or atomic structure. On the other hand, energy-dispersive X-ray spectrometry (EDX) has been used for subtracting compositions in punctual areas, as well as to draw compositional maps of areas ranging from few $\mathrm{nm}^{2}$ up to $\mu \mathrm{m}^{2}$. Those techniques were applied in three STEM microscopes, supplied by Thermo Fisher Scientific: a double-aberration corrected TITAN ${ }^{3}$ Themis and a Talos F200X, both managed by the Central Services of Research, Science and Technology (SC-ICYT, by its initials in Spanish) of the University of Cádiz, and a Talos F200S microscope equipped with Super-X system which includes two silicon drift detectors, which belong to the Research, Technology and Innovation Center of the University of Seville (CITIUS). All three of these microscopes were operated at a $200 \mathrm{kV}$ accelerating voltage.

To observe the samples under the electron beam, the chemically obtained NPs were deposited in holey and/or lacey carbon films on copper TEM grids. For that purpose, 
NP powders were first dissolved in water at different concentrations or even directly deposited on the grid, with the idea of obtaining the optimal particle concentration for TEM observation. To avoid an excessive amount of magnetic NPs in the grid later entered in the TEM microscope (which uses magnetic field-based lenses), a magnet was moved close to the NP-containing grid before its insertion in the microscope sample holder.

\section{Results and discussion}

\section{Structural characterization}

Depending on the dissolution degree with which the NP powders have been deposited on the TEM grids, it is possible to observe conglomerates of NP of different sizes, as it is presented in Fig. 2, besides isolated NPs. In the case of NPs with magnetic cores (Fig. 2b, d), conglomerates are more frequently detected than in the other cases, due to the magnetic forces among those NPs. Nevertheless, in all cases, it was possible to resolve a statistically significant number of particles to carry out size and compositional quantifications. In this sense, as presented in Table 1, it was possible to determine that the fabrication methods lead to average NP sizes lower than $10 \mathrm{~nm}$. The lower size corresponds to GSH-capped CdTe NP, S1, which explains the difficulty for distinguishing among those NPs in low-magnification images, such as the one presented in Fig. 2a. Note that the carbon supporting structure ("C-support", in the image), due to its amorphous atomic arrangement, should not appear in
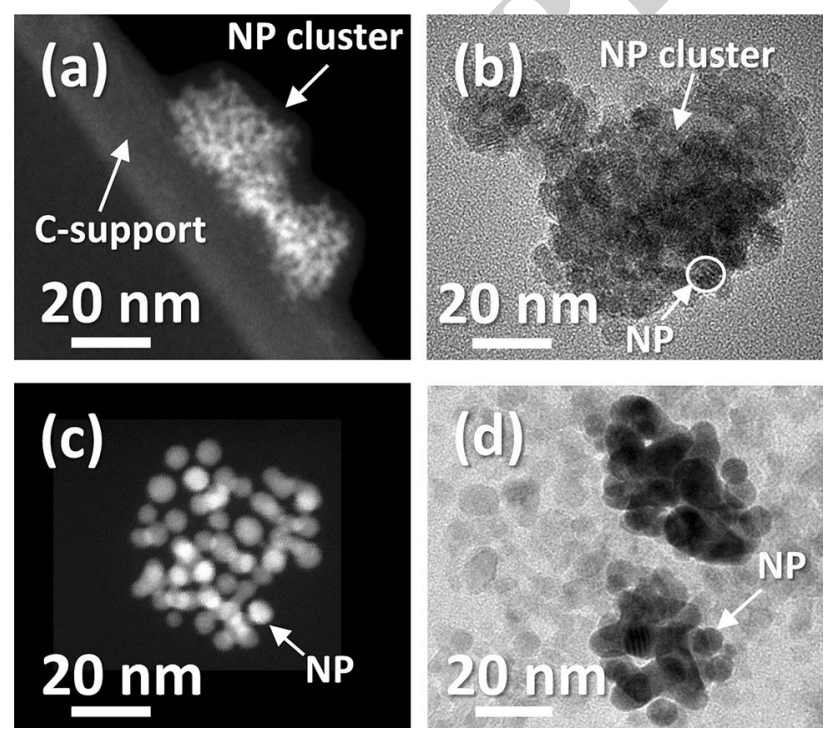

Fig. 2 Accumulations of NPs in samples S1(a), S2(b), S3(c) and S4(d). Images for S1 and S3 correspond to STEM-HAADF mode, and images for $\mathrm{S} 2$ and $\mathrm{S} 4$ are taken in BF-TEM mode
Table 1 NPs and their core average sizes and GSH maximum thicknesses

\begin{tabular}{lllll}
\hline Sample & NP core & NP size $(\mathrm{nm})$ & Core size $(\mathrm{nm})$ & $\begin{array}{l}\text { GSH max. } \\
\text { thickness } \\
(\mathrm{nm})\end{array}$ \\
\hline $\mathrm{S} 1$ & $\mathrm{CdTe}$ & $3.9 \pm 1.1$ & $\mathrm{n} . \mathrm{m}$. & $\mathrm{n} . \mathrm{m}$. \\
$\mathrm{S} 2$ & $\mathrm{Fe}_{3} \mathrm{O}_{4}$ & $7.4 \pm 1.0$ & $5.0 \pm 0.3$ & $0.4 \pm 0.1$ \\
$\mathrm{~S} 3$ & $\mathrm{Au}$ & $5.1 \pm 0.7$ & $5.1 \pm 0.9$ & $2.4 \pm 0.3$ \\
$\mathrm{~S} 4$ & $\mathrm{Fe}_{3} \mathrm{O}_{4} @ \mathrm{Au}$ & $6.5 \pm 1.1$ & $4.4 \pm 1.0$ & $1.1 \pm 0.3$ \\
\hline
\end{tabular}

n.m. not measured

HAADF images since it does not produce electron beam diffraction. Nevertheless, it is not uncommon if brightness level is high enough, for these supporting structures to appear in STEM images. In all cases, rounded and spherical-shaped NPs seemed to be predominant, even more in those samples using gold (samples S3 and S4, Fig. 2c, d), although observations using HRTEM, as it will be commented next in the text, reveal cubic-shaped NP crystalline cores, as it was expected.

The phase-contrast TEM mode, also known as high-resolution TEM or HRTEM, reveals areas with a crystalline structure, such as the NPs cores, by presenting atomic columns or fringes, depending on how the crystalline planes diffract the electronic beam emitted by the microscope source. If the material under observation is amorphous, as it is the case of the GSH shell and the carbon matrix supporting the NPs, these images would show a continuous region where the intensity is distributed without any particular order. This allowed (1) to observe the crystalline nature of the NP core and its size [which is an important property of the particle (Kumari et al. 2019)] and (2) to determinate the maximum thickness of the GSH forming the cap layer in these systems. This is also possible thanks to the use of lacey carbon-type grids, in which particles are frequently found at the border of the carbon-web, and thus the carbon signal does not screen the one from the amorphous NP cover along all the particle surface. With this in mind, it was possible to complete Table 1, indicating also the average size of the core in the nanoparticles and the maximum amorphous glutathione cover thickness, for each studied sample. As a complement to this table, Fig. 3 shows the histograms for the measurements of the NP size. Although the standard deviation in the measurements of these sizes is similar, as it can be observed in Table 1, the difficulty in distinguishing the CdTe QDs (due to the fact that these particles were found in sample $\mathrm{S} 1$ forming clusters or accumulations and not so much isolated) is reflected in the histogram in Fig. 3a, from which it is clear that a lower number of NPs were measured in sample $\mathrm{S} 1$. As expected, the histogram closer to a normal distribution corresponds to the Au NPs (sample S3) since their synthesis is better controlled and it does not present

\footnotetext{
Springer
}

\begin{tabular}{|l|l|l|l|l}
\hline Journal : Large 13204 & Article No : 1418 & Pages : 7 & MS Code : 1418 & Dispatch : 24-4-2020 \\
\hline
\end{tabular}



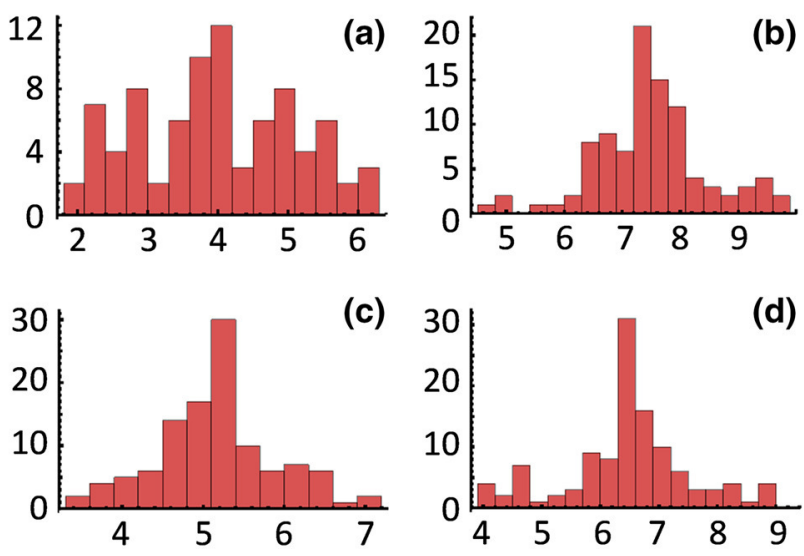

Fig. 3 Histograms for NP sizes in samples S1(a), S2(b), S3(c), and $\mathrm{S} 4(\mathbf{d})$. The horizontal axes indicate the NP size, in $\mathrm{nm}$, while the vertical ones present the amount of NPs corresponding to each size range. The histogram bars correspond to increments in the size of $0.3 \mathrm{~nm}$ magnetic behavior that leads to the cluster formations that would make difficult the measurement of the particle size.

Data in Table 1 together with the results from TEM images such as the ones presented in Fig. 2 indicate the NPs in this work could be a good choice as nanoparticle in medicine since the most attractive NPs for biomedical applications are those spherical and with sizes ranging from 2 to $10 \mathrm{~nm}$ (Khan et al. 2018). Many body barriers fall in the sub-10-nm regime, but, on the other hand, NPs smaller than $2 \mathrm{~nm}$ could considerably increase their toxicity.

In this sense, it can be mentioned that an appropriate method, easier than those based on hydrophobic routs, to prepare small soluble and functionalized NPs is to carry out a reduction in aqueous media using a strong reducing agent, like sodium borohydride, in the presence of strong capping ligands to control particle growth. Some advantages of this route reside in the possibility to choose a capping ligand that directly biofunctionalizes the NP or that facilitates its subsequent biofunctionalization (Fratoddi 2018). This route also allows for the use of thiols as capping agents, due to the high affinity of this group for the NP surface(Dehn et al. 2018).

In this work, we have used this $\mathrm{NaBH}_{4}$ reduction reaction, adding both, the capping and the reduction agent in excess to promote the fast nucleation and successive growth to homogenize NP size. Increasing the nucleation step rate, a high proportion of nuclei will be formed before starting the growth step. This enhances the formation of small homogeneous and spherical cores.

Figure 4 illustrates these ideas through HRTEM images for three types of NPs of this work. This figure does not show particles from sample $\mathrm{S} 1$ since it was not possible to achieve high-resolution conditions for NPs with CdTe cores. On the other hand, for the rest of samples, it was

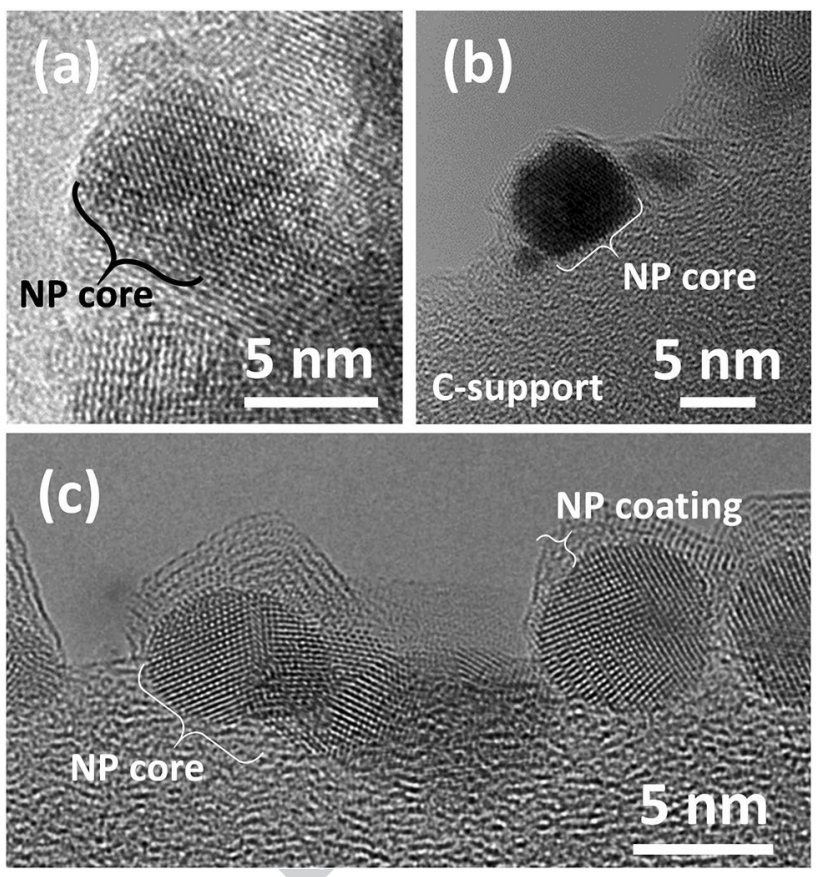

Fig. 4 HRTEM images of individual NPs in samples S2(a), S4(b), and $\mathrm{S} 3(\mathbf{c})$

possible to observe NPs both inside the conglomerates (Fig. 4a) and isolated ones (Fig. 4b, c). Some NP core and coating layers, according with the ideas commented before, are also pointed out in this figure.

It is possible to obtain valuable information from the regions corresponding to the NP core using these HRTEM images. In this sense, the comparison of measurements for atomic planes distances with the ones theoretically expected for the different materials agrees with the affirmation that the NPs cores are formed, indeed, by magnetite and/or gold. In this sense, measurements in images such as the one in Fig. 4a revealed experimental distances of $2.9 \AA$, which agree with the expected distances between $\{220\}$ atomic planes in magnetite particles (Haavik et al. 2000). Figure $4 \mathrm{~b}$ shows an example of a HRTEM image from a NP in sample S4. From this image and other analogous ones, it was possible to measure distances between atomic planes of 3.0 and $4.9 \AA$. This is in agreement with theoretical distances between the $\{220\}$ and $\{111\}$ families of planes of magnetite ( 2.97 and $4.85 \AA$, respectively) (Haavik et al. 2000), and also $2.4 \AA$, which can be associated with the ones for the $\{111\}$ planes in the case of $\mathrm{Au}$ (Righter et al. 2011). The HRTEM images of nanoparticles in Fig. 4d shows atomic distances of 2.1 and $2.4 \AA$, which, respectively, agree well with the spacing between $\{002\}$ and $\{111\}$ families of atomic planes for cubic $\mathrm{Au}$ (2.04 and $2.35 \AA$, respectively) (Righter et al. 2011).

\begin{tabular}{|l|l|l|l|l|}
\hline Journal : Large 13204 & Article No : 1418 & Pages : 7 & MS Code : 1418 & Dispatch : 24-4-2020 \\
\hline
\end{tabular}



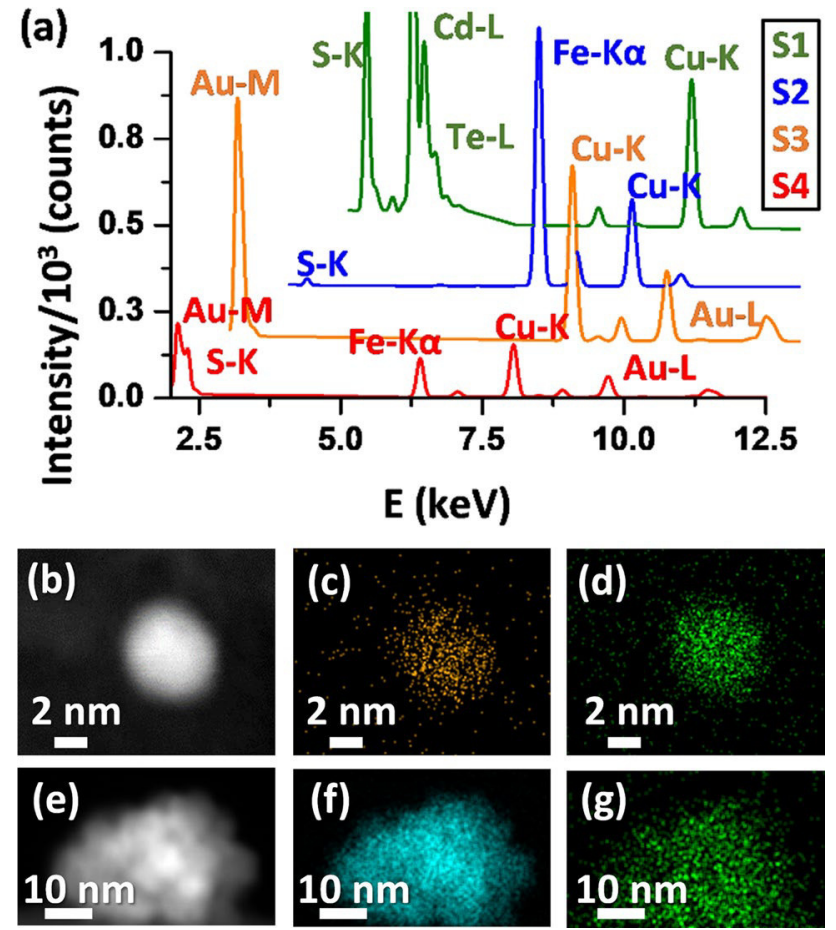

Fig. 5 Punctual EDX spectra from NPs for all samples, with the corresponding element and X-ray peaks and color legend (a). Isolated NP in sample S3: HAADF image (b) and EDX maps for Au (c) and $\mathrm{S}$ (d). Agglomeration of NPs in sample S2: HAADF image (e) and EDX map for $\mathrm{Fe}(\mathbf{f})$ and $\mathrm{S}(\mathrm{g})$

\section{Compositional characterization}

Figure 5 displays representative punctual EDX spectra for NPs in the four samples (Fig. 5a), as well as EDX maps for NPs (Fig. 5b-d) and accumulations of those (Fig. 5e-g). It is possible to observe, in Fig. 5a, that the NPs have, indeed, the expected chemical composition, revealing a sulfur K $\alpha$-peak, at $2.31 \mathrm{keV}$, in all cases (the gold M-peak, at $2.12 \mathrm{keV}$, which appears in samples S3 and S4, partly overlap the sulfur signal). This element is associated with the presence of the glutathione shell around the NP core. Figure $5 \mathrm{~b}$ presents a HAADF image of a single NP (sample S3) for which an EDX map has been recorded. Figure $5 \mathrm{c}$ reveals that this NP has a gold core, while it is clear, according to Fig. 5d, that the NP is totally covered with the S-containing compound (glutathione). In this sense, it is also possible to show that the glutathione coats the NPs even when they form conglomerates. Figure 5e presents a HAADF image of a 30-nm-size NP agglomeration. EDX maps of iron (Fig. 5f) and sulfur (Fig. 5g) reveal that there is a homogeneous distribution of magnetite and GSH, respectively, indicating that the synthesis method leads to a majority of NPs in the sample with the desired core and the glutathione layer. This result is also observed in the other samples.

\section{Conclusions}

A series of samples of GSH-capped NPs have been successfully developed via chemical methods. The smallest and rounder NPs, with the most clearly monocrystalline structure of the core, have been obtained for gold-containing NPs. On the other hand, worse results have been obtained for the NPs with a CdTe core. (S)TEM-based techniques represent a good choice to completely characterize these systems. The spectroscopic techniques, combined with STEM images, are especially indicated to reveal the sulfur in the glutathione layer, which allows the visualization of this NP shell.

Acknowledgements The authors would like to acknowledge the SCICYT and the CITIUS central services of the Universities of Cádiz and Seville, respectively, which made possible the sample preparation and TEM studies in this work. A. J. Santos would like to thank the IMEYMAT Institute and the Spanish Ministry of Education and Culture for the concessions of Grants ICARO-173873 and FPU16-04386. The authors would like to thank the Spanish Ministry of Economy and Competitiveness for the economic support to the research project MAT2015-67354-R (Program "Plan I+D + i", subprogram "Retos").

Author contributions RL and OB-M contributed to the study conception and design. Nanoparticle systems were synthetized by RL and EF. (S)TEM microscopy techniques were carried out at three different microscopes by AMB, JMM, AJS and FMM. OB-M and JMM, as "investigadores principales" (main researchers) of Research Project MAT2015-67,354-R, managed the necessary economical funds that allowed this work. The first draft of the manuscript was written by AMB and JMM and all authors commented on the previous versions of the manuscript. All authors read and approved the final manuscript.

Funding This work has been funded by the Spanish Ministry of Economy and Competitiveness, through the research project "Sintesis asistida por láser de nanopartículas ternarias: una ruta alternativa para la fabricación de nuevos agentes de contraste multimodales para el diagnóstico temprano del cáncer de mama", reference: MAT201567354-R, of the Program "Plan I+D +i", subprogram "Retos".

Data availability Any of the data and material utilized to develop this manuscript can be available at request of any researcher, via the corresponding author.

Code availability Not applicable.

\section{Compliance with ethical standards}

Conflict of interest The authors declare that there is no conflict of interest/competing interests associated with the work presented in this manuscript.

\section{References}

Amendola V, Pilot R, Frasconi M et al (2017) Surface plasmon resonance in gold nanoparticles: a review. J Phys Condens Matter. https://doi.org/10.1088/1361-648X/aa60f3 
Beato-López JJ, Fernández-Ponce C, Blanco E et al (2012) Preparation and characterization of fluorescent $\mathrm{CdS}$ quantum dots used for the direct detection of GsT fusion proteins. Nanomater Nanotechnol. https://doi.org/10.5772/53926

Beato-López JJ, Espinazo ML, Fernández-Ponce C et al (2017) CdTe quantum dots linked to Glutathione as a bridge for protein crosslinking. J Lumin 187:193-200. https://doi.org/10.1016/j. jlumin.2017.03.012

Birrenbach G, Speiser PP (1976) Polymerized micelles and their use as adjuvants in immunology. J Pharm Sci 65:1763-1766. https:// doi.org/10.1002/jps.2600651217

Dehn MH, Arseneau DJ, Buck T et al (2018) Nature of magnetism in thiol-capped gold nanoparticles investigated with Muon spin rotation. Appl Phys Lett. https://doi.org/10.1063/1.5017768

Fernández-Ponce C, Muñoz-Miranda JP, de los Santos DM et al (2018) Influence of size and surface capping on photoluminescence and cytotoxicity of gold nanoparticles. J Nanoparticle Res. https://doi. org/10.1007/s11051-018-4406-0

Fratoddi I (2018) Hydrophobic and hydrophilic Au and Ag nanoparticles. Breakthroughs and perspectives. Nanomaterials. https://doi. org/10.3390/nano8010011

Gheibi Hayat SM, Darroudi M (2019) Nanovaccine: a novel approach in immunization. J Cell Physiol 234:12530-12536. https://doi. org/10.1002/jcp. 28120

Griffin S, Masood MI, Nasim MJ et al (2018) Natural nanoparticles: a particular matter inspired by nature. Antioxidants. https://doi. org/10.3390/antiox7010003

Haavik C, Stølen S, Fjellvåg H et al (2000) Equation of state of magnetite and its high-pressure modification: thermodynamics of the Fe-O system at high pressure. Am Mineral 85:514-523. https:// doi.org/10.2138/am-2000-0413

Hochella MF (2002) There's plenty of room at the bottom: nanoscience in geochemistry. Geochim Cosmochim Acta 66:735-743. https:// doi.org/10.1016/S0016-7037(01)00868-7

Hong R, Han G, Fernández JM et al (2006) Glutathione-mediated delivery and release using monolayer protected nanoparticle carriers. J Am Chem Soc 128:1078-1079. https://doi.org/10.1021/ ja056726i

Kairdolf BA, Smith AM, Stokes TH et al (2013) Semiconductor quantum dots for bioimaging and biodiagnostic applications. Annu Rev Anal Chem 6:143-162. https://doi.org/10.1146/annurev-anche m-060908-155136

Karley D, Gupta D, Tiwari A (2011) Biomarker for cancer: a great promise for future. World J Oncol 2:151-157. https://doi. org/10.4021/wjon.v2i4.352

Khan HA, Sakharkar MK, Nayak A et al (2018) Nanoparticles for biomedical applications: an overview. Elsevier Ltd., Amsterdam

Kreuter J (2009) Guest editorial: special issue for Prof. Dr. Peter Paul Speiser. J Drug Target 17:562-563. https://doi.org/10.1080/10611 860903090337
Kumar A, Huo S, Zhang X et al (2014) Neuropilin-1-targeted gold nanoparticles enhance therapeutic efficacy of platinum(IV) drug for prostate cancer treatment. ACS Nano 8:4205-4220. https:// doi.org/10.1021/nn500152u

Kumari Y, Kaur G, Kumar R et al (2019) Gold nanoparticles: new routes across old boundaries. Adv Colloid Interface Sci 274:102037. https://doi.org/10.1016/j.cis.2019.102037

Matsui I (2005) Nanoparticles for electronic device applications: a brief review. J Chem Eng Japan 38:535-546. https://doi.org/10.1252/ jcej.38.535

Ramalingam V (2019) Multifunctionality of gold nanoparticles: plausible and convincing properties. Adv Colloid Interface Sci 271:101989. https://doi.org/10.1016/j.cis.2019.101989

Righter K, Sutton S, Danielson L et al (2011) The effect of fO2 on the partitioning and valence of $\mathrm{v}$ and $\mathrm{Cr}$ in garnet/melt pairs and the relation to terrestrial mantle $\mathrm{v}$ and $\mathrm{Cr}$ content. Am Mineral 96:1278-1290. https://doi.org/10.2138/am.2011.3690

Le Trequesser Q, Seznec H, Delville MH (2013) Functionalized nanomaterials: their use as contrast agents in bioimaging: mono- and multi-modal approaches. Nanotechnol Rev 2:125-169. https://doi. org/10.1515/ntrev-2012-0080

Ventola CL (2017) Progress in nanomedicine: approved and investigational nanodrugs. PT 42:742-755

Zhang Z, Huo F, Zhang X, Guo D (2012) Fabrication and size prediction of crystalline nanoparticles of silicon induced by nanogrinding with ultrafine diamond grits. Scr Mater 67:657-660. https:// doi.org/10.1016/j.scriptamat.2012.07.016

Zhang Z, Guo D, Wang B et al (2015) A novel approach of high speed scratching on silicon wafers at nanoscale depths of cut. Sci Rep 5:1-9. https://doi.org/10.1038/srep16395

Zhang Z, Cui J, Wang B et al (2017) A novel approach of mechanical chemical grinding. J Alloys Compd 726:514-524. https://doi. org/10.1016/j.jallcom.2017.08.024

Zhang Z, Cui J, Zhang J et al (2019) Environment friendly chemical mechanical polishing of copper. Appl Surf Sci 467-468:5-11. https://doi.org/10.1016/j.apsusc.2018.10.133

Zhang Z, Liao L, Wang X et al (2020) Development of a novel chemical mechanical polishing slurry and its polishing mechanisms on a nickel alloy. Appl Surf Sci 506:144670. https://doi.org/10.1016/j. apsusc. 2019.144670

Publisher's Note Springer Nature remains neutral with regard to jurisdictional claims in published maps and institutional affiliations. 